\title{
Spreading properties of a multi-Gaussian Schell-model vortex beam in slanted atmospheric turbulence
}

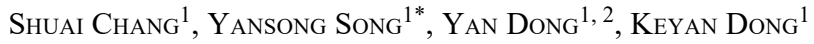 \\ ${ }^{1}$ National and Local Joint Engineering Research Center of Space Optoelectronics Technology, \\ Changchun University of Science and Technology, Changchun, 130022, China \\ ${ }^{2}$ School of Electronics and Information Engineering, Changchun University of Science and Technology, \\ Changchun 130022, China
}

*Corresponding author: songyansong2018@sohu.com

\begin{abstract}
The cross-spectral density function of a multi-Gaussian Schell-model vortex (MGSMV) beam propagating through slanted atmospheric turbulence was derived, and the influences of the MGSMV beam parameter and slanted atmospheric turbulence on the spreading properties of a MGSMV beam are studied. One can find that a MGSMV beam propagating in slanted atmospheric turbulence can evolve into the flat-topped beam, and a MGSMV beam with larger index $N$ and topological charge $M$ propagating in slanted atmospheric turbulence will lose the dark hollow center and evolve into the Gaussian beam more slowly than the MGSMV beam with smaller index $N$ and topological charge $M$. It is also found that a MGSMV beam propagating in slanted atmospheric turbulence with larger strucutre parameter $C$ will evolve into Gaussian beam faster, but the influences of zenith angle $\alpha$ on the spreading properties of MGSMV beam in the far field can be ignored.
\end{abstract}

Keywords: average intensity, vortex beam, multi-Gaussian Schell-model source, atmospheric turbulence, slanted path.

\section{Introduction}

With the development of the free-space optical communications, the propagation properties of laser beam in turbulent medium have attracted the attention of many researchers in past years. Until now, the influences of turbulent medium on the propagation properties of various beams have been studied, such as partially coherent beam [1], random electromagnetic multi-Gaussian Schell-model vortex beam [2] , vortex beam [ $\underline{3}$ ], four -petal Gaussian beams [4] , partially coherent four-petal Gaussian vortex beams []ㅡ, flat-topped vortex hollow beam [6], partially coherent crescent-like optical beam [7], radial phased-locked partially coherent anomalous hollow beam array []], spectrally 
partially coherent Gaussian-Schell model pulsed beam [9], array beam [10-12], partially coherent anomalous elliptical hollow Gaussian beam [13], Airy beam [14], partially coherent electromagnetic Gaussian-Schell model pulse beams [15], flattened -vortex beam [16], partially coherent Hermite-Gaussian beam [17], $J(0)$-correlated partially coherent beam [18], single photon [19], Laguerre-Gaussian beam [20], general multi-Gaussian beam [21] , twisted rectangular multi-Gaussian Schell-model beam [22], radially polarized multi-cosine Gaussian-Schell model beam [23], multi-Gaussian Schell-model vortex beam [24], and square multi-Gaussian Schell-model beam [25], etc. However, the propagation properties of a multi-Gaussian Schell-model vortex (MGSMV) beam propagating in slanted atmospheric turbulence has not been reported. In this paper, the cross-spectral density function of a MGSMV beam propagating through slanted atmospheric turbulence was derived, and the spreading properties of a MGSMV beam propagating in slanted atmospheric turbulence were investigated.

\section{Propagation of a MGSMV beam in slanted atmospheric turbulence}

In the Cartesian coordinate system, the laser beam propagating along the $z$-axis, the cross-spectral density function of a MGSMV beam at the source plane $L=0$ can be expressed as $[\underline{24}, \underline{26}]$

$$
\begin{aligned}
W\left(\mathbf{r}_{10}, \mathbf{r}_{20}, 0\right) \\
=\frac{1}{C_{0}^{2}} \exp \left(-\frac{x_{10}^{2}+y_{10}^{2}}{w^{2}}-\frac{x_{20}^{2}+y_{20}^{2}}{w^{2}}\right)\left(x_{10}+i y_{10}\right)^{M}\left(x_{20}-i y_{20}\right)^{N} \\
\quad \times \sum_{n=1}^{N}\left(\begin{array}{l}
N \\
n
\end{array}\right) \frac{(-1)^{n-1}}{n} \exp \left[-\frac{\left(x_{10}-x_{20}\right)^{2}}{2 n \sigma^{2}}-\frac{\left(y_{10}-y_{20}\right)^{2}}{2 n \sigma^{2}}\right]
\end{aligned}
$$

with

$$
C_{0}=\sum_{n=1}^{N}\left(\begin{array}{l}
N \\
n
\end{array}\right) \frac{(-1)^{n-1}}{n}
$$

where $W\left(\mathbf{r}_{10}, \mathbf{r}_{20}, 0\right)$ is the cross-spectral density function of a MGSMV beam at the source plane $L=0, \mathbf{r}_{0}=\left(x_{0}, y_{0}\right)$ is the position vector at the source plane $L=0, N$ is the beam index of multi-Gaussian Schell-model source, $M$ is the topological charge, and $\sigma$ are the spatial coherence length.

Based on the extended Huygens-Fresnel diffraction principle, the cross-spectral density function of a partially coherent beam propagating through slanted atmospheric turbulence at the propagation distance $L$ can be expressed as [믄 20$]$ 


$$
\begin{aligned}
& W\left(\mathbf{r}_{1}, \mathbf{r}_{2}, L\right) \\
& =\frac{k^{2}}{4 \pi^{2} z^{2}} \int_{-\infty}^{+\infty} \int_{-\infty}^{+\infty} \int_{-\infty}^{+\infty} \int_{-\infty}^{+\infty} W\left(\mathbf{r}_{10}, \mathbf{r}_{20}, 0\right) \exp \left[-\frac{i k}{2 z}\left(\mathbf{r}_{1}-\mathbf{r}_{10}\right)^{2}+\frac{i k}{2 z}\left(\mathbf{r}_{2}-\mathbf{r}_{20}\right)^{2}\right] \\
& \quad \times\left\langle\exp \left[\psi\left(\mathbf{r}_{10}, \mathbf{r}_{1}\right)+\psi^{*}\left(\mathbf{r}_{20}, \mathbf{r}_{2}\right)\right]\right\rangle \mathrm{d} \mathbf{r}_{10} \mathrm{~d} \mathbf{r}_{20}
\end{aligned}
$$

with

$$
\begin{aligned}
& \left\langle\exp \left[\psi\left(\mathbf{r}_{10}, \mathbf{r}_{1}\right)+\psi^{*}\left(\mathbf{r}_{20}, \mathbf{r}_{2}\right)\right]\right\rangle \\
& \quad=\exp \left[-\frac{\left(\mathbf{r}_{10}-\mathbf{r}_{20}\right)^{2}+\left(\mathbf{r}_{10}-\mathbf{r}_{20}\right)\left(\mathbf{r}_{1}-\mathbf{r}_{2}\right)+\left(\mathbf{r}_{1}-\mathbf{r}_{2}\right)^{2}}{\rho_{0}^{2}}\right]
\end{aligned}
$$

where $\rho_{0}$ is the coherence length of a spherical wave propagating in slanted atmospheric turbulence, and which can be expressed as

$$
\rho_{0}=\left[1.46 k^{2} \int_{0}^{L} C_{n}^{2}(z \cos \alpha)\left(1-\frac{z}{L}\right) \mathrm{d} z\right]^{-3 / 5}
$$

where $C_{n}^{2}(z \cos \alpha)$ is the refractive index structure parameter of slanted atmospheric turbulence, and $\alpha$ represents the zenith angle. The $C_{n}^{2}(z \cos \alpha)$ can be expressed as $[\underline{27}, \underline{28}]$

$$
\begin{aligned}
C_{n}^{2}(z \cos \alpha)= & 8.148 \times 10^{-56} v^{2}(z \cos \alpha)^{10} \exp \left(-\frac{z \cos \alpha}{1000}\right) \\
& +2.7 \times 10^{-16} \exp \left(-\frac{z \cos \alpha}{1500}\right)+C \exp \left(-\frac{z \cos \alpha}{100}\right)
\end{aligned}
$$

where $v=2.1 \mathrm{~m} / \mathrm{s}$ is the rms wind speed, $C$ is the structure parameter of atmosphere turbulence at the ground.

Submitting Eq. (1) into Eq. (3), and considering the following integral equations [29]:

$$
\begin{aligned}
& (x+i y)^{M}=\sum_{l=0}^{M} \frac{M ! i^{l}}{l !(M-l) !} x^{M-l} y^{l} \\
& \int_{-\infty}^{+\infty} x^{n} \exp \left(-p x^{2}+2 q x\right) \mathrm{d} x \\
& \quad=n ! \exp \left(\frac{q^{2}}{p}\right)\left(\frac{q}{p}\right)^{n} \sqrt{\frac{\pi}{p}} \sum_{k=0}^{\left[\frac{n}{2}\right]} \frac{1}{k !(n-2 k) !}\left(\frac{p}{4 q^{2}}\right)^{k}
\end{aligned}
$$




$$
H_{2 d}(x)=\sum_{l=0}^{d} \frac{(-1)^{l}(2 d) !}{l !(2 d-2 l) !}(2 x)^{2 d-2 l}
$$

The cross-spectral density function of a MGSMV beam propagating in slanted atmospheric turbulence can be derived as

$$
\begin{aligned}
W\left(\mathbf{r}_{1}, \mathbf{r}_{2}, L\right) & \\
= & \frac{k^{2}}{4 \pi^{2} z^{2}} \exp \left[-\frac{i k}{2 z}\left(x_{1}^{2}+y_{1}^{2}\right)+\frac{i k}{2 z}\left(x_{2}^{2}+y_{2}^{2}\right)\right] \\
& \times \exp \left[-\frac{\left(x_{1}-x_{2}\right)^{2}+\left(y_{1}-y_{2}\right)^{2}}{\rho_{0}^{2}}\right] \frac{1}{C_{0}^{2}} \sum_{n=1}^{N}\left(\begin{array}{c}
N \\
n
\end{array}\right) \frac{(-1)^{n-1}}{n} \\
& \times \sum_{l_{1}=0}^{M} \frac{M ! i^{l_{1}}}{l_{1} !\left(M-l_{1}\right) !} \sum_{l_{2}=0}^{M} \frac{M !(-i)^{l_{2}}}{l_{2} !\left(M-l_{2}\right) !} W(x, L) W(y, L)
\end{aligned}
$$

where

$$
\begin{aligned}
& W(x, L) \\
& =\left(M-l_{1}\right) ! \sqrt{\frac{\pi}{a_{x}}}\left(\frac{1}{a_{x}}\right)^{M-l_{1}} \exp \left[\frac{1}{a_{x}}\left(\frac{i k}{2 z} x_{1}-\frac{x_{1}-x_{2}}{2 \rho_{0}^{2}}\right)^{2}\right] \\
& \times\left[\frac{M-l_{1}}{\sum_{k=0}^{2}} \frac{1}{k !\left(M-l_{1}-2 k\right) !}\left(\frac{a_{x}}{4}\right)^{k} \sum_{s=0}^{M-l_{1}-2 k} \frac{\left(M-l_{1}-2 k\right) !}{s !\left(M-l_{1}-2 k-s\right) !}\right. \\
& \times\left(\frac{i k}{2 z} x_{1}-\frac{x_{1}-x_{2}}{2 \rho_{0}^{2}}\right)^{M-l_{1}-2 k-s}\left(\frac{1}{2 n \sigma^{2}}+\frac{1}{\rho_{0}^{2}}\right)^{s} \sqrt{\frac{\pi}{b_{x}}} \\
& \times 2^{-\left(M-l_{2}+s\right)} i^{M-l_{2}+s} \exp \left(\frac{c_{x}^{2}}{b_{x}}\right)\left(\frac{1}{b_{x}}\right)^{0.5\left(M-l_{2}+s\right)} H_{M-l_{2}+s}\left(-\frac{i c_{x}}{\sqrt{b_{x}}}\right)
\end{aligned}
$$




$$
\begin{aligned}
& W(y, L) \\
& =\left(l_{1}\right) ! \sqrt{\frac{\pi}{a_{y}}}\left(\frac{1}{a_{y}}\right)^{l_{1}} \exp \left[\frac{1}{a_{y}}\left(\frac{i k}{2 z} y_{1}-\frac{y_{1}-y_{2}}{2 \rho_{0}^{2}}\right)^{2}\right] \\
& \quad \times \sum_{k=0}^{\left[\frac{l_{1}}{2}\right]} \frac{1}{k !\left(l_{1}-2 k\right) !}\left(\frac{a_{y}}{4}\right)^{k} \sum_{s=0}^{l_{1}-2 k} \frac{\left(l_{1}-2 k\right) !}{s !\left(l_{1}-2 k-s\right) !} \\
& \times\left(\frac{i k}{2 z} y_{1}-\frac{y_{1}-y_{2}}{2 \rho_{0}^{2}}\right)^{l_{1}-2 k-s}\left(\frac{1}{2 n \sigma^{2}}+\frac{1}{\rho_{0}^{2}}\right)^{s} \sqrt{\frac{\pi}{b_{y}}} \\
& \times 2^{-\left(l_{2}+s\right)} i^{l_{2}+s} \exp \left(\frac{c_{y}^{2}}{b_{y}}\right)\left(\frac{1}{b_{y}}\right)^{0.5\left(l_{2}+s\right)} H_{l_{2}+s}\left(-\frac{i c_{y}}{\sqrt{b_{y}}}\right)
\end{aligned}
$$

with

$$
\begin{aligned}
& a_{\gamma}=\frac{1}{w^{2}}+\frac{1}{2 n \sigma^{2}}+\frac{1}{\rho_{0}^{2}}+\frac{i k}{2 z}, \quad \gamma=x, y \\
& b_{\gamma}=\frac{1}{w^{2}}+\frac{1}{2 n \sigma^{2}}+\frac{1}{\rho_{0}^{2}}-\frac{i k}{2 z}-\frac{1}{a_{\gamma}}\left(\frac{1}{2 n \sigma^{2}}+\frac{1}{\rho_{0}^{2}}\right)^{2} \\
& c_{\gamma}=\frac{\gamma_{1}-\gamma_{2}}{2 \rho_{0}^{2}}-\frac{i k}{2 z} \gamma_{2}+\frac{1}{a_{\gamma}}\left(\frac{i k}{2 z} \gamma_{1}-\frac{\gamma_{1}-\gamma_{2}}{2 \rho_{0}^{2}}\right)\left(\frac{1}{2 n \sigma^{2}}+\frac{1}{\rho_{0}^{2}}\right)
\end{aligned}
$$

The intensity of a MGSMV beam propagating in slanted atmospheric turbulence at the receiver plane $L$ can be expressed as

$$
I(\mathbf{r}, L)=W(\mathbf{r}, \mathbf{r}, L)
$$

\section{Numerical results and analyses}

In this section, the spreading properties of a MGSMV beam propagating in slanted atmospheric turbulence are studied by using the equations derived in the above section. The parameters of MGSMV beam and slanted atmospheric turbulence are chosen as $w=1 \mathrm{~cm}, \lambda=532 \mathrm{~nm}, M=1, N=5, \sigma=5 \mathrm{~mm}, C=1.5 \times 10^{-14} \mathrm{~m}^{-2 / 3}$ and $\alpha=\pi / 4$ without other explanations in captions of following figures. 
a
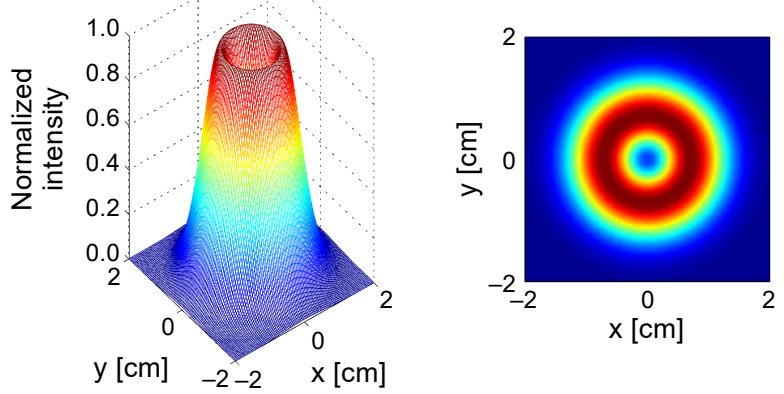

b
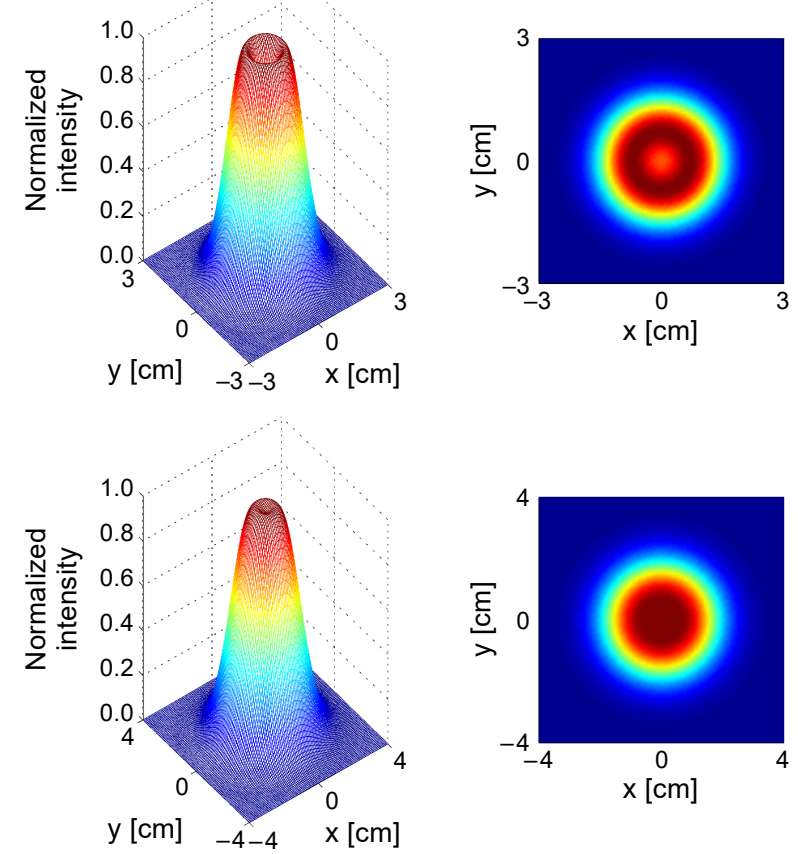

d
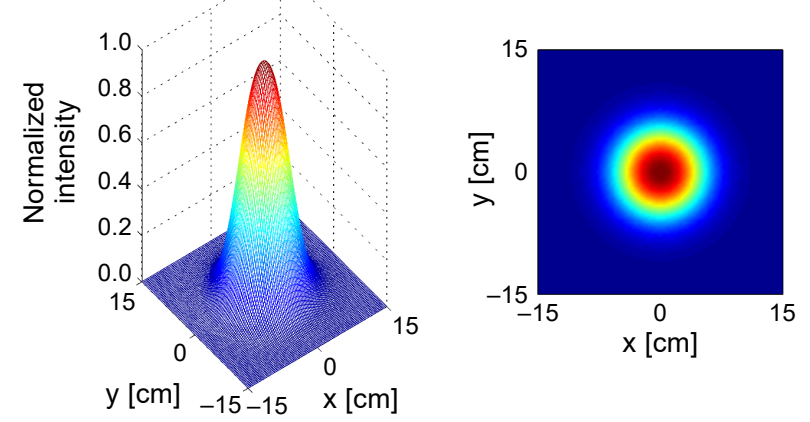

Fig. 1. Three-dimensional normalized intensity of a MGSMV beam propagating in slanted atmospheric turbulence: $z=100 \mathrm{~m}(\mathbf{a}), z=300 \mathrm{~m}(\mathbf{b}), z=500 \mathrm{~m}(\mathbf{c})$, and $z=2000 \mathrm{~m}(\mathbf{d})$. 
The 3D normalized average intensity of a MGSMV beam propagating in slanted atmospheric turbulence is illustrated in Fig. 1. From Fig. 1, one can find that the MGSMV beam propagating in slanted atmospheric turbulence at the short propagation distance can keep the initial dark hollow center produced by the $M$ (Fig. 1a); the dark hollow center will disappear during the propagation distance increasing (Fig. 1b). At a certain propagation distance, the MGSMV beam will evolve into a flat-topped beam (Fig. 1c), and this phenomenon is introduced by the multi-Gaussian Schell-model source. At last, the MGSMV beam will evolve into the Gaussian beam in the far field (Fig. 1d). From previous reports [28], it is also found that the flat-topped beam propagating in turbulent media will evolve into the Gaussian beam in the far field.

To investigate the influences of MGSMV beam parameters $N, M$ and $\sigma$ on the spreading of a MGSMV beam propagating in slanted atmospheric turbulence, the cross-sections of normalized intensity of a MGSMV beam propagating in slanted atmospheric turbulence at different propagation distance for different $N, M$ and $\sigma$ are given in Figs. 2-4, respectively. From Fig. 4, one can find that the MGSMV beam with larger $N$ will lose the dark hollow center and evolve into the flat-topped beam more slowly than
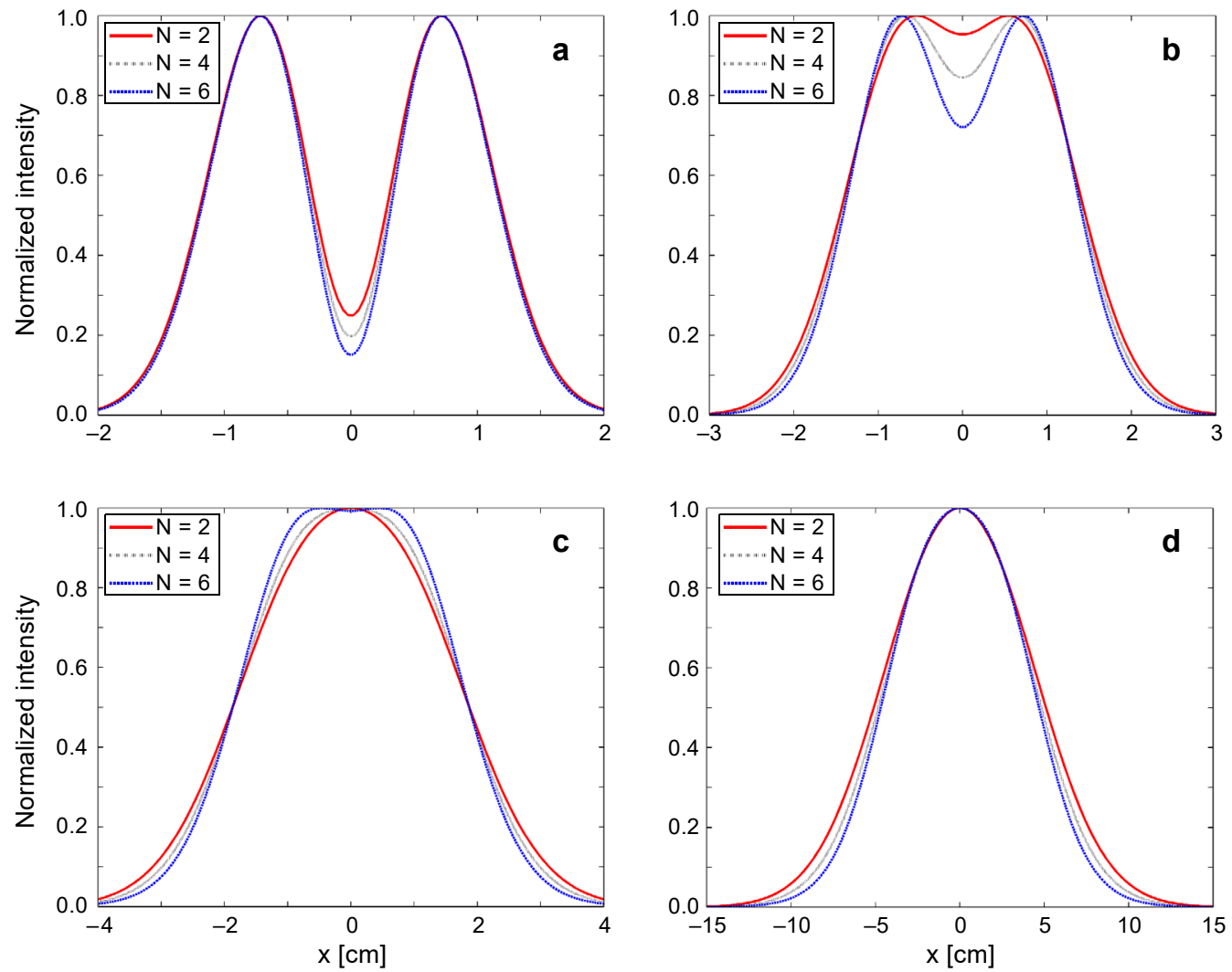

Fig. 2. Cross-sections of normalized intensity of a MGSMV beam propagating in slanted atmospheric turbulence for different $N ; z=100 \mathrm{~m}(\mathbf{a}), z=300 \mathrm{~m}(\mathbf{b}), z=600 \mathrm{~m}(\mathbf{c})$, and $z=2000 \mathrm{~m}(\mathbf{d})$. 

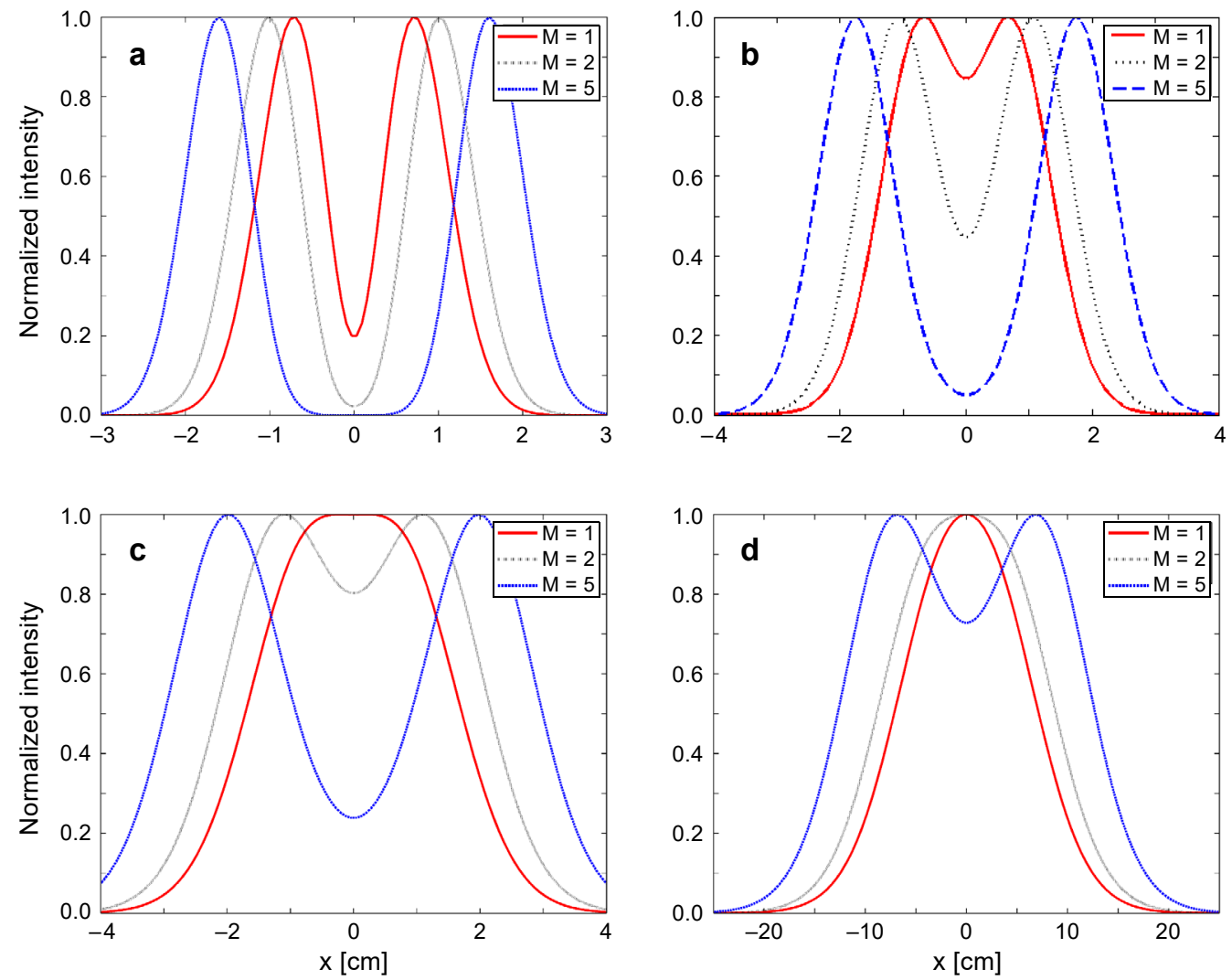

Fig. 3. Cross-sections of normalized intensity of a MGSMV beam with $N=4$ propagating in slanted atmospheric turbulence for different $M ; z=100 \mathrm{~m}(\mathbf{a}), z=300 \mathrm{~m}(\mathbf{b}), z=500 \mathrm{~m}(\mathbf{c})$, and $z=3000 \mathrm{~m}(\mathbf{d})$.
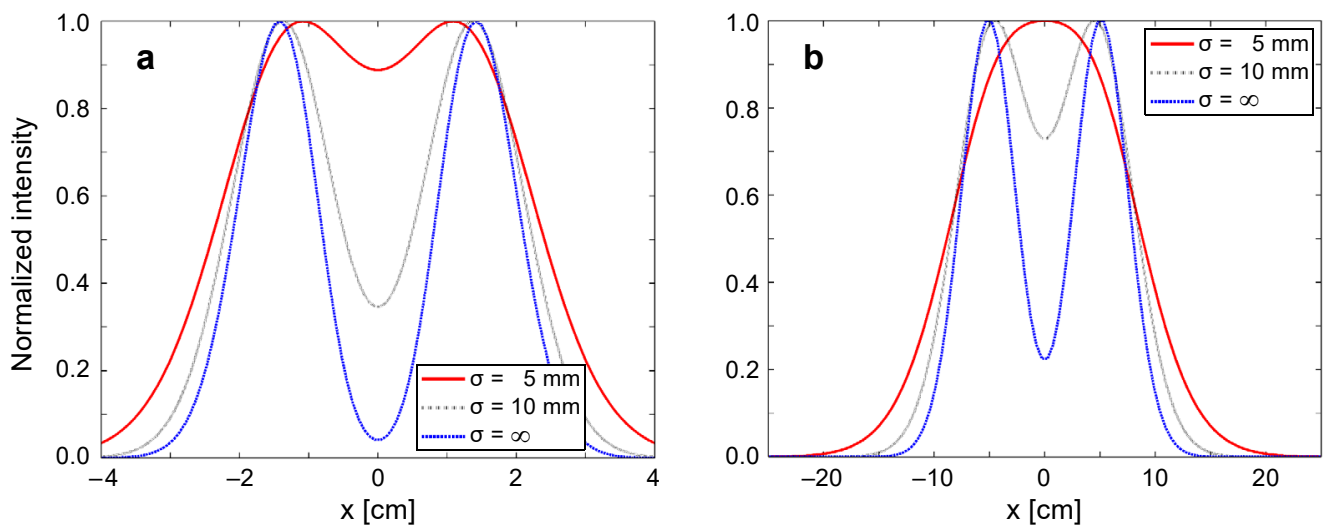

Fig. 4. Cross-sections of normalized intensity of a MGSMV beam with $N=4$ and $M=2$ propagating in slanted atmospheric turbulence for different $\sigma ; z=600 \mathrm{~m}(\mathbf{a})$, and $z=3000 \mathrm{~m}(\mathbf{b})$. 
the MGSMV beam with smaller $N$ (Figs. $2 \mathbf{b}$ and $2 \mathbf{c}$ ). As the propagation distance increases, the MGSMV beam with different $N$ will all evolve into the Gaussian beam, and the beam with smaller $N$ will evolve into the Gaussian beam and spread faster, but the beam with different $N$ will have the similar beam spot in the far field (Fig. 2d). It is found from Fig. 3 that the MGSMV beam with large $M$ will have a larger beam spot with a larger dark hollow center (Fig. 3a). As the propagation distance increases, the MGSMV beam with smaller $M$ will first lose the initial beam profile and evolve into the flat-topped beam (Fig. 3b); and the MGSMV beam with the larger $M$ will gradually evolve into flat-topped beam and the Gaussian beam as the propagation distance increases (Fig. 3d). In the studies of $\sigma$ on the spreading of the MGSMV beam, it is found that the MGSMV beam with smaller coherence length $\sigma$ will evolve into the flat-topped beam faster than the beam with larger coherence length and fully coherence beam (Fig. 4b).

In order to study the influences of slanted atmospheric turbulence parameters $C$ and $\alpha$ on the spreading of a MGSMV beam propagating in slanted atmospheric turbulence, Figs. 5 and 6 show the cross-sections of normalized intensity of a MGSMV beam propagating in slanted atmospheric turbulence for different $C$ and $\alpha$, respectively. From Fig. 5, it is found that a MGSMV beam propagating in slanted atmospheric turbulence with larger $C$ will lose the initial dark hollow and evolve into Gaussian beam faster, and the beam propagating in slanted atmospheric turbulence with smaller $C$ will also evolve into the flat-topped beam (Fig. 5b). In the studies of the influences of the zenith angle $\alpha$ on spreading properties, it is found that a MGSMV beam propagating in slanted atmospheric turbulence at the same propagation distance with smaller $\alpha$ will lose the dark hollow center more rapidly, but the influences of the zenith angle $\alpha$ is not an evidence (Fig. 6a). In the far field, one can find that a MGSMV beam propagating in slanted atmospheric turbulence for different $\alpha$ at the same propagation distance will have the same beam spot (Fig. 6b). Thus, the influences of the zenith angle $\alpha$ on the
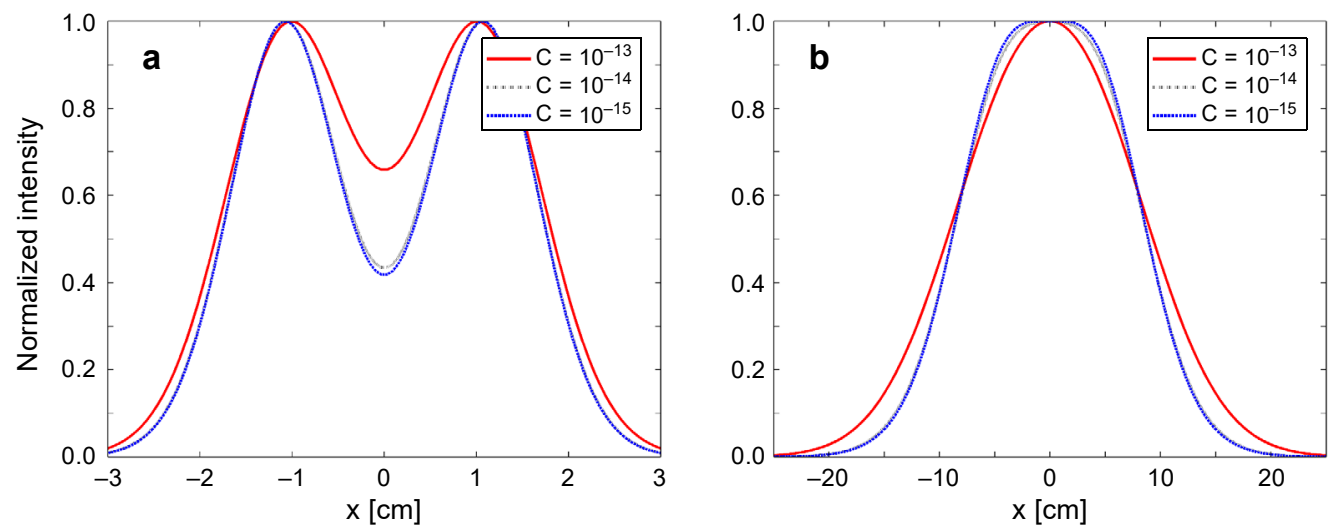

Fig. 5. Cross-sections of normalized intensity of a MGSMV beam propagating in slanted atmospheric turbulence for different $C ; z=300 \mathrm{~m}(\mathbf{a})$, and $z=3000 \mathrm{~m}(\mathbf{b})$. 

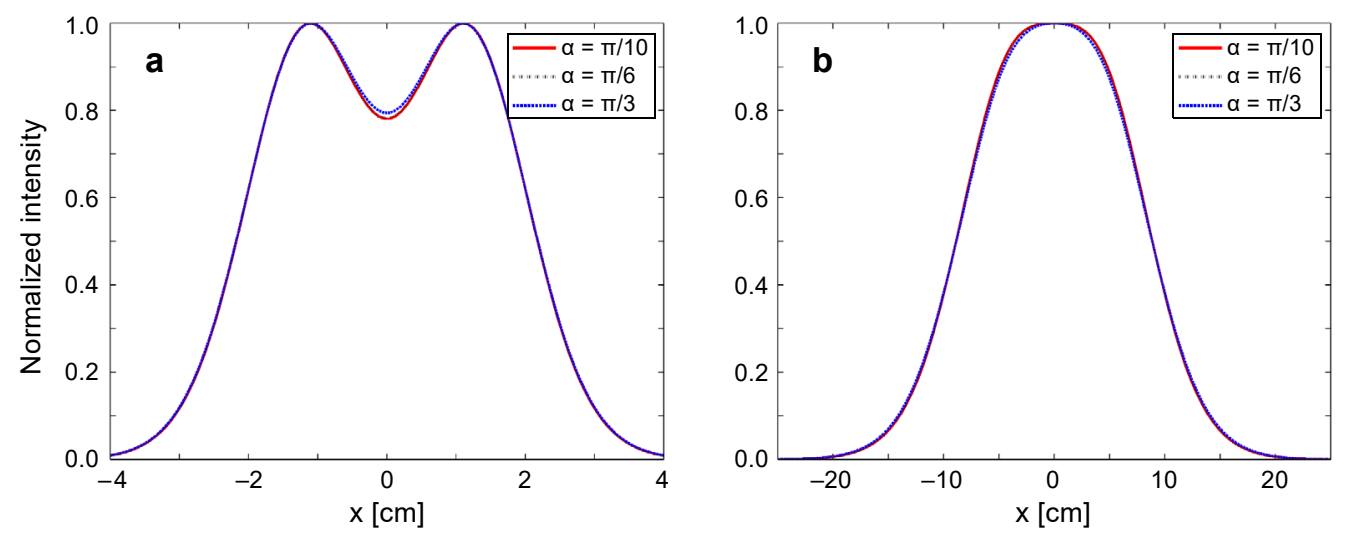

Fig. 6. Cross-sections of normalized intensity of a MGSMV beam propagating in slanted atmospheric turbulence for different $\alpha ; z=500 \mathrm{~m}(\mathbf{a})$, and $z=3000 \mathrm{~m}(\mathbf{b})$.

MGSMV beam can be ignored in the far field, because it is different from the other beam propagating in slant atmosphere [30].

\section{Conclusions}

In this paper, the spreading properties of a MGSMV beam propagating in slanted atmospheric turbulence have been analyzed by using numerical examples. It is found that a MGSMV beam propagating in slanted atmospheric will lose the initial dark hollow center and evolve into a flat-topped beam as the propagation distance increases; and the MGSMV beam with larger $N$ and $M$ will lose the dark hollow center and evolve into the Gaussian beam more slowly than the MGSMV beam with smaller $N$ and $M$. It is also found that a MGSMV beam propagating in slanted atmospheric turbulence with larger $C$ will lose the initial dark hollow and evolve into the Gaussian beam faster. However, the influences of the zenith angle $\alpha$ on the spreading properties of the MGSMV beam in the far field can be ignored.

Acknowledgments - This work was supported by the Natural Science Foundation of Jilin Province (Grant No. 20180101031JC) and the Jilin Provincial Research Foundation for Basic Research (Grant No. 2019C040-7).

\section{References}

[1] Wang F., Liu X., CAi Y., Propagation of partially coherent beam in turbulent atmosphere: a review (invited review), Progress in Electromagnetics Research 150, 2015, pp. 123-143, DOI: 10.2528/PIER 15010802.

[2] Liu D., WAng Y., Properties of a random electromagnetic multi-Gaussian Schell-model vortex beam in oceanic turbulence, Applied Physics B 124, 2018, article 176, DOI: 10.1007/s00340-018-7048-0.

[3] Li N., Chu X., Zhang P., Feng X., Fan C., Qiao C., Compensation for the orbital angular momentum of a vortex beam in turbulent atmosphere by adaptive optics, Optics and Laser Technology 98, 2018, pp. 7-11, DOI: 10.1016/j.optlastec.2017.07.028. 
[4] Liu D., Wang Y., Yin H., Average intensity of four-petal Gaussian beams through paraxial optical system in atmosphere turbulence, Optik 127(6), 2016, pp. 3225-3229, DOI: 10.1016/j.ijleo.2015.12.084.

[5] Liu D., Wang Y., Yin H., Propagation properties of partially coherent four-petal Gaussian vortex beams in turbulent atmosphere, Optics and Laser Technology 78, 2016, pp. 95-100, DOI: 10.1016/ j.optlastec.2015.10.004.

[6] LiU D., WANG Y., WANG G., YIN H., Intensity properties of flat-topped vortex hollow beams propagating in atmospheric turbulence, Optik 127(20), 2016, pp. 9386-9393, DOI: 10.1016/j.ijleo.2016. $\underline{07.026 .}$.

[7] Wang F., Li J., Martinez-Piedra G., Korotkova O., Propagation dynamics of partially coherent crescent-like optical beams in free space and turbulent atmosphere, Optics Express 25(21), 2017, pp. 26055-26066, DOI: 10.1364/OE.25.026055.

[8] Wang K.L., Zhaо C.H., Propagation properties of a radial phased-locked partially coherent anomalous hollow beam array in turbulent atmosphere, Optics and Laser Technology 57, 2014, pp. 44-51, DOI: 10.1016/j.optlastec.2013.09.037.

[9] Liu D., Luo X., Wang G., WAng Y., Spectral and coherence properties of spectrally partially coherent Gaussian Schell-model pulsed beams propagating in turbulent atmosphere, Current Optics and Photonics 1(4), 2017, pp. 271-277.

[10] LiU D., WANG Y., Evolution properties of a radial phased-locked partially coherent Lorentz-Gauss array beam in oceanic turbulence, Optics and Laser Technology 103, 2018, pp. 33-41, DOI: 10.1016/ j.optlastec.2018.01.014.

[11] Liu D., WAng Y., Zhong H., Average intensity of radial phased-locked partially coherent standard Hermite-Gaussian beam in oceanic turbulence, Optics and Laser Technology 106, 2018, pp. 495-505, DOI: $10.1016 /$ j.optlastec.2018.05.015.

[12] Liu H.L., LÜ Y.F., XIA J., Chen D., He W., Pu X.Y., Radial phased-locked partially coherent flat -topped vortex beam array in non-Kolmogorov medium, Optics Express 24(17), 2016, pp. 19695 -19712, DOI: 10.1364/OE.24.019695.

[13] Tian H.H., Xu Y.G., Yang T., Ma Z.R., Wang S.J., Dan Y.Q., Propagation characteristics of partially coherent anomalous elliptical hollow Gaussian beam propagating through atmospheric turbulence along a slant path, Journal of Modern Optics 64(4), 2017, pp. 422-429, DOI: 10.1080/09500340. 2016.1241441.

[14] Yin X., Zhang L.C., Quantum polarization fluctuations of an Airy beam in turbulent atmosphere in a slant path, Journal of the Optical Society of America A 33(7), 2016, pp. 1348-1352, DOI: 10.1364 / JOSAA.33.001348.

[15] Gao M., Li Y., Lv H., Gong L., Polarization properties of polarized and partially coherent electromagnetic Gaussian-Schell model pulse beams on slant path in turbulent atmosphere, Infrared Physics and Technology 67, 2014, pp. 98-106, DOI: 10.1016/j.infrared.2014.06.008.

[16] Wu H.Y., Li X., Sheng S., Huang Z.S., Huang S.H., Zhao S.Q., Wang H., Sun Z.H., Xu X.G., Properties of the flattened-vortex beam with aperture propagating through the turbulent atmosphere in a slant path, Optical Engineering 52(7), 2013, article 077105, DOI: 10.1117/1.OE.52.7.077105.

[17] Zhang L.C., Yin X., Zhu Y., Polarization fluctuations of partially coherent Hermite-Gaussian beams in a slant turbulent channel, Optik 125(13), 2014, pp. 3272-3276, DOI: 10.1016/j.ijleo.2013. $\underline{12.045}$.

[18] CANG Ji, Zhang Yi-XIN, The propagation properties of J0-correlated partially coherent beams in the slant atmosphere, Acta Physica Sinica-Chinese Edition 58(4), 2009, pp. 2444-2450, DOI: 10.7498/aps.58.2444.

[19] Zhang Y.-X., WANG Y.-G., XU J.-C., WANG J.-Y., JIA J.-J., Orbital angular momentum crosstalk of single photons propagation in a slant non-Kolmogorov turbulence channel, Optics Communications 284(5), 2011, pp. 1132-1138, DOI: 10.1016/j.optcom.2010.10.077.

[20] Ke XI-Zheng, Chen JuAn, YAng Yi-Ming, Study on orbital angular momentum of Laguerre-Gaussian beam in a slant-path atmospheric turbulence, Acta Physica Sinica-Chinese Edition 63(15), 2014, article 150301, DOI: 10.7498/aps.63.150301. 
[21] Chu X.X., Liu Z.J., Wu Y., Propagation of a general multi-Gaussian beam in turbulent atmosphere in a slant path, Journal of the Optical Society of America A 25(1), 2008, pp. 74-79, DOI: 10.1364/ JOSAA.25.000074.

[22] Zноч Y.J., Zнао D.M., Propagation properties of a twisted rectangular multi-Gaussian Schell-model beam in free space and oceanic turbulence, Applied Optics 57(30), 2018, pp. 8978-8983, DOI: $10.1364 / \mathrm{AO} .57 .008978$.

[23] TANG M., Zhao D., Li X., WANg J., Propagation of radially polarized multi-cosine Gaussian Schell -model beams in non-Kolmogorov turbulence, Optics Communications 407, 2018, pp. 392-397, DOI: 10.1016/j.optcom.2017.09.067.

[24] Wang X., Yao M., Yi X., Qiu Z., Liu Z., Spreading and evolution behavior of coherent vortices of multi-Gaussian Schell-model vortex beams propagating through non-Kolmogorov turbulence, Optics and Laser Technology 87, 2017, pp. 99-107, DOI: 10.1016/j.optlastec.2016.08.003.

[25] Zhang H., Fu W., Polarization properties of square multi-Gaussian Schell-model beam propagating through non-Kolmogorov turbulence, Optik 134, 2017,pp. 161-169, DOI: 10.1016/j.ijleo.2017.01.045.

[26] Zhang Y.T., Liu L., Zhao C.L., Cai Y.J., Multi-Gaussian Schell-model vortex beam, Physics Letters A 378(9), 2014, pp. 750-754, DOI: 10.1016/j.physleta.2013.12.039.

[27] Dou L., Ji X., Li P., Propagation of partially coherent annular beams with decentered field in turbulence along a slant path, Optics Express 20(8), 2012, pp. 8417-8430, DOI: 10.1364/OE.20.008417.

[28] Xu J.-C., Zhang Y.-X., WANG J.-Y., JIA J.-J., Detection probability of single photons in a slant path atmospheric turbulence communication channel, Optik 122(7), 2011, pp. 586-590, DOI: 10.1016/ j.ijleo.2010.04.015.

[29] JEFFrey A., Hui DAI, Handbook of Mathematical Formulas and Integrals, 4th Ed., Academic Press, 2008.

[30] Dong K.Y., Dong Y., Song Y.S., Chang S., The properties of anomalous hollow beam propagating in the slant atmosphere, Optik 172, 2018, pp. 1040-1046, DOI: 10.1016/j.ijleo.2018.07.127. 